\title{
Approach to the Diagnosis of Bleeding Disorders in Children
}

\author{
Tawfique M
}

\begin{abstract}
Summary
Hemostasis is the cessation of bleeding in the intravascular compartment. This occurs by the formation of clot formation at the site of injury. The intricately related system also regulates the size of the clots by the activation of fibrinolysis. The disorders that lead to the bleeding outside the intravascular space is bleeding disorder. Bleeding occurs as a result of defective quality or quantitative deficiencies of platelets, coagulation problems in the extrinsic or intrinsic or the common pathways and abnormalities in the vascular walls to contain hemorrhage. Although the values of different coagulation and fibrinlytic factors are pretty much like that of the adults yet, neonates have considerable differences in these values than their senior counterparts even in pediatric age groups. Considering all these facts the history, physical examination and laboratory investigations always provide the clinicians some important clues to reach the diagnosis. Because of the complicated relationship among the different pathways of coagulations and fibrinolytic systems the whole procedure might look complex yet methodical approach paves the way of an intelligent clinician to arrive at the diagnosis with precision and clinical perfection.
\end{abstract}

CBMJ 2020 July: vol. 09 no. 02 P: 45-53

\section{Introduction}

Recognizing excessive bleeding and treating bleeding disorders may be challenging. Awareness of the differential, diagnostic strategies and treatment options is critical. $^{1}$ Hemostasis is the active process that clots blood in areas of blood vessel injury yet simultaneously limits the clot size only to the areas of injury. Over time, the clot is lysed by the fibrinolytic system and normal blood flow is restored. The main components of the hemostatic process are the vessel walls, platelets, coagulation proteins and fibrinolytic system. ${ }^{2}$ Having all these factors involved in the whole system it appears more frequently than naught to resolve the clinical puzzles of bleeding disorders in pediatrics. That is why the issue was addressed here, to make the evaluation of the bleeding children look easy.

\section{The overview of Hemostatic System:}

\section{Hemostasis:}

Cessation of bleeding occurs within the intravascular compartment lined with endothelium. Normal hemostasis and thrombosis involve a number of factors, including platelets, granulocytes and monocytes as well as the coagulation, fibrinolytic and anticoagulant protein systems. Each of the three protein systems balances the activities of the others. In addition, the integrity of the vessel wall, which in the intravascular compartment is lined by endothelium, is constitutively an anticoagulant surface. When injured, either physically or by an inflammatory state, the endothelium can be a locus for procoagulant and antifibrinolytic activity.

\section{Components:}

The physiologic hemostatic system has two limbs.

a. The first is a large group of proteins that participate in clot formation (coagulation).

b. The second is a cell component that consists mostly of platelets but also includes granulocytes, monocytes and endothelial cells.

\section{Regulation:}

Physiologic hemostasis is a tightly regulated balance between the formation and dissolution of hemostasis plugs. The coagulation system is a group of proteins that participate in clot formation. The

1. Dr. Muhammad Tawfique, Consultant, Pediatrics and Pediatric Hematology and Oncology, Bangladesh Specialized Hospital.

Address of correspondence:

Email: mtawfique@gmail.com 
Fibrinolhytic system is a group of proteins that participate in clot dissolution. The anticoagulation system is a group of proteins that regulate the coagulation and fibrinolytic systems. The proteins of the anticoagulation system join those of the fibrinolytic system to prevent or counterbalance coagulation reactions. Thus, the hemostatic system is tightly modulated by a series of enzymes and scaffolding proteins. ${ }^{3}$

\section{The process of Hemostasis:}

Hemostasis is a process to prevent and stop bleeding meaning to keep blood within a damaged blood vessel. It is the first stage of wound healing. This involves coagulation. Intact blood vessels are the ones that regulates clot formation inside a vessel. ${ }^{4}$ The endothelium and its cells lining the intact vessels prevent blood clotting with a heparin-like molecule and thromobodulin and prevent platelet aggregation by the help of Nitric Oxide and Prostacyclin. When endothelial injury occurs, the endothelial cells stop secretion of coagulation and aggregation inhibitors and instead secrete Von Willibrand Factor (VWF), which initiate the maintenance of hemostasis after injury. Hemostasis has three major steps: 1) vasoconstriction, 2) temporary blockage of a break by a platelet plug, and 3) blood coagulation, or formation of a fibrin clot. These processes seal the hole until tissues are repaired. $^{5}$

Hemostasis occurs when blood is present outside the body or blood vessels. It is the innate response for the body to stop bleeding and loss of blood. As shown in Figure $I^{6}$ during hemostasis three steps occur in a rapid sequence. Vascular spasm is the first response as the blood vessels constrict to allow less blood to be lost. In the second step, platelets stick together to form a temporary seal to cover the break in the vessel wall. The third and last step is called coagulation or blood clotting. Coagulation reinforces the platelet plug with fibrin threads that act as a "molecular glue". ${ }^{7}$ Within seconds of disruption of epithelial wall of blood vessels, platelets begin to adhere to the sub endothelial surface. It takes approximately sixty seconds until the first fibrin strands begin to intersperse among the wound. After several minutes the platelet plug is completely formed by fibrin. ${ }^{8}$ Hemostasis is maintained in the body via three mechanisms:

1. Vasoconstriction - Vasoconstriction is produced by vascular smooth muscle cells, and is the blood vessel's first response to injury. The smooth muscle cells of vascular endothelium releases intravascular signals to control the contracting properties. When a blood vessel is damaged, there is an immediate reflex, initiated by local sympathetic pain receptors, which helps promote vasoconstriction. The damaged vessels will constrict (vasoconstrict) which reduces the amount of blood flow through the area and limits the amount of blood loss. Collagen is exposed at the site of injury, the collagen promotes platelets to adhere to the injury site. Platelets release cytoplasmic granules which contain serotonin, ADP and thromboxane $\mathrm{A} 2$, all of which increase the effect of vasoconstriction. The more the damage the more is response and the more the effects the more smaller the blood vessels are. ${ }^{9,10}$

2. Platelet plug formation- Platelets adhere to damaged endothelium to form a platelet plug (primary hemostasis) and then degranulate. This process is regulated through thromoboregulation. Plug formation is activated by VWF, available abundantly in plasma in a healthy human being. Platelets play a major role in the hemostatic process. When platelets come in contact with injured endothelial cells, they change shape, release granules and ultimately 'sticky'. Platelets express certain receptors, some of which are used for the adhesion of platelets to collagen. When platelets are activated, they express glycoprotein receptors that interact with other platelets, producing aggregation and 
adhesion. Platelets release cytoplasmic granules such as ADP, serotonin and TXA2. ADP attracts more platelets to the affected area while serotonin is a vasoconstrictor and thromboxane A2 assists in platelet aggregation, vasoconstriction and degranulation. As more chemicals are released more platelets stick and release their chemicals; creating a platelet plug and continuing the process in a positive feedback loop. Platelets alone are responsible for stopping the bleeding of unnoticed wear and tear of our skin on a daily basis. This is referred to as primary hemostasis. ${ }^{9,11}$

3. Cot formation - Once the platelet plug has been formed by the platelets, the clotting factors are activated in a sequence of events known as 'coagulation cascade' that leads to the formation of Fibrin from inactive fibrinogen plasma protein. Thus, a Fibrin mesh is produced all around the platelet plug to hold it in place; this step is called "Secondary Hemostasis". During this process some red and white blood cells are trapped in the mesh resulting in the more integrity of the plug formation as the resultant plug is called as 'thrombus' or 'Clot'. Therefore, 'blood clot' contains secondary hemostasis plug with blood cells trapped in it. Though this clot formation is the essential one for wound healing yet the kind of overactivities of clotting process might bring about the serious health hazards like stroke, ishchemic heart diseases and pulmonary embolism ${ }^{8}$.

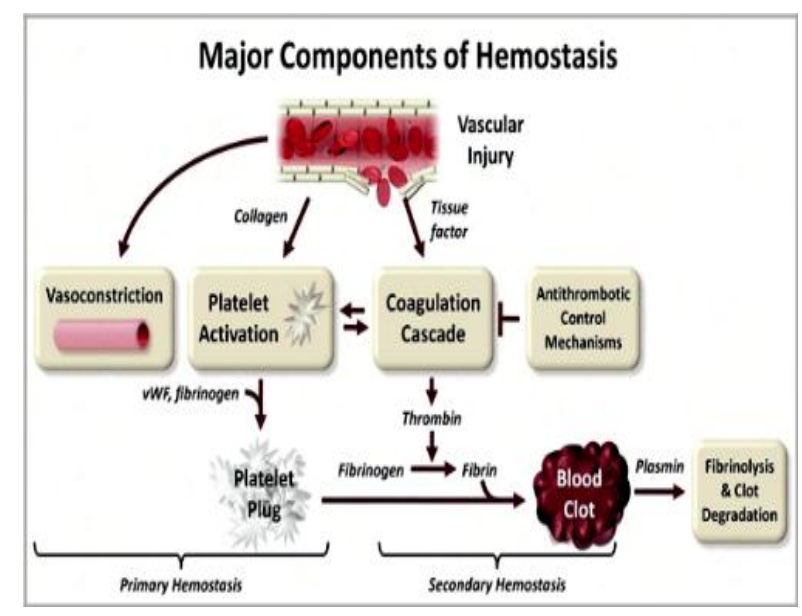

Figure 1: Schematic presentation of Homeostasis. ${ }^{6}$

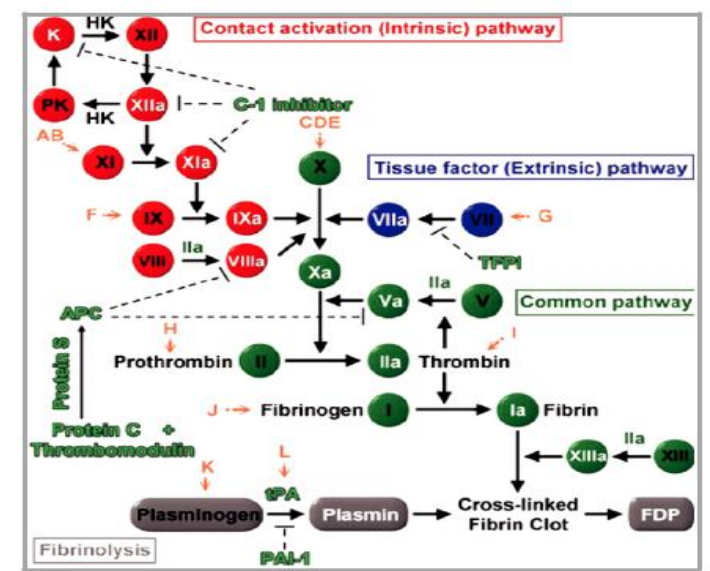

Figure 2: Schematic presentation of Coagulation Cascade. $^{12}$

\section{A brief coagulation cascade and the coagulation pathways:}

Figure 2 here depicted here the activation of both the intrinsic and extrinsic pathways and formation of thrombin and then fibrin meshworks to consolidate the platelet plugs and thrombus. It also shows the activation of fibrinolytic systems like formation of Kallikrein, Plasmin and the roles of Protein $S$ and Protein $C$ to cause fibrinolysis and regulate the thrombus formation in the way to maintain the hemostasis. ${ }^{12}$

\section{Overview of classification of bleeding disorders:}

1. Bleeding due to Platelet disorder:
a. Platelet deficiency
b. Platelet dysfunction

2. Bleeding due to Coagulopathy:
a. Clotting factor deficiency
b. Presence of inhibitors

3. Bleeding due to Vascular causes

\section{Investigations of bleeding disorders: Initial screening tests: ${ }^{13,13 a}$}

1. Complete Blood Count (CBC): Here the quantitative assessment of platelets and review of blood smear to assess platelet morphology could give one the clue to detect thrombocytopenia and some of the structural defects of the platelets relating to platelet dysfunction. 


\section{Assessment of platelet function:}

a) Platelet Function Analyzer (PFA-100): Assesses flow through a membrane, membrane closure time is measured in response to ADP and epinephrine. The closure time is often prolonged with impaired platelet function.

b) Bleeding time is not used as a standard test because of the difficulties in standardization. In our country BT is used as PFA-100 is not known to be available.

3. Coagulation factor screening tests:

a. The coagulation system is divided into the Intrinsic, the extrinsic and common pathways. However the division is more conceptual than physiological.

4. Prothrombin time assay: Assesses the function of extrinsic pathway. This test is done by using thromboplastin and calcium chloride to set the formation of thrombin.

5. Activated Partial Thromboplastin time: The test estimates the Intrinsic Pathway. This test utilizes a phospholipid reagent to set off the onset of the enzyme reaction that leads to the formation of thrombin via the Intrinsic Pathway

Common Confirmatory Coagulation Assays: ${ }^{13,13 a}$

- Fibrinogen assay: Quantitative measurement of Fibrinogen, useful when both the PT and aPTT are prolonged.

- Thrombin time: Prolonged when fibrinogen is reduced or abnormal, in the presence of inhibitors, FDPs, D-dimers and in the presence of thrombin - inhibiting drugs as well as hypoalbuminemia.

- The reptilase time is a modification of the TT, in which Reptilase is used instead of thrombin and the test is unaffected by heparin and heparin like anticoagulants.
- Mixing studies- In cases PT or aPTT being prolonged this is test is useful by adding normal plasma to patient's plasma. A normal result indicates a clotting factor deficiency and persistence of prolongation of values suggests presence of an inhibitor of a clotting factor.

- Clotting factor activity assays are performed to detect the factor deficiencies as mixing study comes out normal. If aPTT was prolonged and gets normalized in mixing study FXII, FXI, and FVIII assays are indicated. When PT only was prolonged and becomes corrected in mixing study FVII assay should be done. As both the PT and aPTT were prolonged and mixing study shows normal values FX, FV, FII and Fibrinogen studies are likely to be conclusive.

- Von Willebrand antigen: Quantitative assay for VWF, useful when PFA-100 closure time is prolonged or when VWD is suspected.

- VWF multimers are useful when discrepancies between VWF antigen (normal to low) and ristocetin cofactor activity (extremely low; ratio of ristocetin cofactor activity to VWF antigen $<0.6$ ).

- Platelet aggregation studies: This is a qualitative assessment of platelet function, considered useful when platelet function disorders are suspected.

- Urea clot lysis assay: Not a very sensitive test. The test is done as a screening for FXIII deficiency outside the newborn period.

Global Hemostatic Tests: ${ }^{13,13 a}$

Global Hemostatic Tests can provide detailed information on thrombin generation and processes downstream including fibrin polymerization and fibrin dissolution.

- Thrombin Generation Assay: The calibrated automated Thrombogram (CAT) system uses a fluorogenic substrate to continuously measure the generated thrombin. The endogenous 
thrombin potential (eTP), which can be measured by calculating the area under the cure from the throbogram, has shown correlation with the bleeding phenotypes in hemophilia, in hemophilia, patients with inhibitors and factor XI deficiency.

- Thromboelastography (TEG): TEG is performed on whole blood, assessing the viscoelastic property of clot formation under low shear condition after the addition of specific coagulation activators. Improvement in the methodology is widely expanding the use of TEG from preclinical research settings to point of care resting in intensive care units and operating rooms. The device has a metal pin suspended by a torsion wire immersed into a cup which holds the whole blood. Once clotting starts, fibrin strands formed increase the torque between the pin and the cup which is measured electronically. TEG provides various data relating to clot formation and fibrinolysis (the lag time before the clot starts to form, the rate at which clotting occurs, the maximal amplitude of the trace or clot strength, and the extent and rate of amplitude). ${ }^{13,13 a}$

\section{Special Considerations for Bleeding in Neonates:}

With the reference to current evidence based knowledge the human hemostatic system is highly influenced by the age. Certainly the levels and values of the platelets, coagulation factors, inhibitors are affected by the maternal condition, strains and stresses related to birth processes and the variables of the neonates being born and growing through early infancy. ${ }^{14}$ The understanding about these variations is very important to appreciate the individual newborns and infants and to evaluate their variability. The data in neonatal patients and infancy should also be analyzed from different perspective to evaluate the clinical conditions and for overall management. ${ }^{15-18}$

The reference ranges of PT, APTT, TT vary in neonates significantly depending on their age.
The coagulation proteins are unable not cross placental barriers. These are the proteins synthesized by the fetus at the 10th week of gestation and onwards. ${ }^{19}$ The absolute values as well as the relative ones of coagulation proteins in the neonate differ from the adult values and are dependent to varying extent on both the gestational and postnatal age of the infant. The Vit $\mathrm{K}$ dependent factors II, VII, IX X are almost $50 \%$ of the adult values in term infants at birth. This might not be the same in preterm varying variably in pre terms. After birth these factors build up in concentration not to a definite pattern.

Usually Factor VII levels reach the adult range by 5 days. The other factors take six months to rise to adult values. The contact factors like factors XI, XII, Prekallikrein and High Molecular Weight Kininongen are $30-50 \%$ of normal at term. This is not so in case of Factor VIII that is found at the same level as in adults and remain so through the neonatal period. On the other hand VWF levels are higher at birth and although the level declines slightly yet remains high compared with adult values until around 3 months. However, multimers of VWF are unusually large which are very unlike of older children. $^{20,21}$ The stronger aggregation of platelets in response to Ristocetin proved these multimers being more active functionally. ${ }^{22}$ As compared to adults the newborns at birth have the factor XIII $70 \%$ of normal increasing to adult levels by day five. Because of the fact that factor VIII level is normal at birth it is possible to diagnose Hemophilia $A$ in this age group regardless of its severity. The same is not true in case of Von Willebr and disease where diagnosis should not be attempted before the age of six months. Of commonly used screening tests the PT is only minimally prolonged in normal term infants and shortens within the first month of life. This contrasts with the APTT which can be markedly prolonged particularly in the preterm infants. ${ }^{15}$

There are some direct inhibitors direct inhibitors like antithrombin III (ATIII), Heparin Cofactor $\mathrm{H}$ $(\mathrm{HCH})$ and alpha 2 Macroglobulin ( $\alpha 2 \mathrm{M})$. 
ATIII and HTH are around $50 \%$ of normal at birth gradually increasing to reach adult levels by around 3 and 6 months respectively. In contrast $\alpha 2 \mathrm{M}$ is increased at birth and continues to rise postnatally with levels at 6 months which are approximately twice the normal adult values. Protein $\mathrm{C}$ and Protein $\mathrm{S}$ are Vitamin $\mathrm{K}$ dependent inhibitors acting indirectly and are both measured $<50 \%$ of normal at birth, which simulates the reduction in the Vit $\mathrm{K}$ dependent procoagulants. Although concentrations of both proteins increase after birth, mean protein $\mathrm{C}$ concentrations remain significantly low during infancy and not reaching adult concentrations until the early teenage years. ${ }^{23,24}$ This is pertinent to mention that $\mathrm{C} 4$ binding protein (C4b) is absent in neonates. As a result Protein $S$ circulates entirely in its active free form. ${ }^{25.26}$ Available data indicates that Tissue Factor Pathway Inhibitor (TFPI) or extrinsic pathway inhibitor (EPI) are reduced in cord plasma at birth to around $65 \%$ of adult values. ${ }^{27,28}$

Similarly the fibrinoytic system is not as active as adult normal values due to the lower level of plasminogen and $\alpha 2 \mathrm{AP}$ being $50 \%$ and $80 \%$ respectively at birth compared to adult normal values. $^{29}$

\section{Approach to diagnosis of bleeding disorders:}

Like all the clinical problems the approach to diagnosis of bleeding disorders certainly demands the clinical evaluation in the forefront followed by the lab investigations like screening first and then the specific factor or inhibitor assays.

Clinical evaluation requires to know the history and physical examination findings. In history one should know first the type and site of bleeding, the onset, the duration, spontaneous or related to any trauma, type of healing, any associated symptoms like fever, or evidence of sepsis or inflammations, family history of similar nature, extended family members being affected with bleeding disorders, consanguinity between the parents and in neonates any maternal drug or disease. By evaluating history spontaneous bleeding and prolonged bleeding even after trauma always indicate a bleeding problem. The congenital causes of bleeding like some platelet deficiencies might start very early in infancy. In newborn period there can be some serious bleeding due to Congenital Afibrinogenemia or dysfibrinogenemia or Hemophilias and Hemorrhagic Disease of Newborn. The petichae and purpura like exclusively cutaneous bleeding are likely due to Platelet disorders and deep seated or mucosal bleeding are usually due to coagulopathies like Hemophilias. However, VWD causes mucosal or more often bleeding in the skin and usually with presentation of Hematomas. Here, Family history or extended family history should give clinicians the important clues to diagnose the hereditary causes of bleeding., ${ }^{2,13 a}$

The physical examination could also provide the clinicians critical clues like infections relating the bleeding to sepsis and DIC, inflammations and arthritis relating to Connective Tissue disorders, lymphadenopathy and hepatosplenomegaly due to malignancies like Acute Leukemias or occasionally suggesting for Infectious Mononeucleosis or DIC or Connective Tissue Disorders.

In absence of any other physical findings except the bleeding only the cutaneous bleeding suggests for Platelet related causes like ITP, bone marrow dysfunction or platelet dysfunction, or platelet adhesion abnormality like VWD. If the findings were only deep seated bleeding like limb hematoma or hemarthroses that indicated for factor deficiencies like Hemophilias, Afibrinogenemia, dysfibrinogenemia and presence of inhibitors in blood. ${ }^{2,13 a}$

Keeping all these facts in mind a clinician should proceed on to plan for the investigations like CBC, PT, APTT and Bleeding Time first and then for the others like TT, Mixing studies, platelet function tests, d-dimer. Finally assay of the specific factors and inhibitors should confirm 
the diagnosis. However, with suggestions from history, physical findings a patient with petichae or with mucosal bleeding might require a Bone Marrow study to confirm the suspicion of Bone Marrow dysfunction or Hypoplasia or Hypersplenism and ITPs. Certainly Bone Marrow study must be confirmatory of Leukemias and other Bone Marrow infiltrative disorders and rarely storage diseases resulting in thrombocytopenia and bleeding. ${ }^{2,13 a}$

- History \& Exam

- $\quad$ CBC, Plate let, PT, PTT, Fibinogen, TT, BT, vWF

- Platelets $\downarrow$

- $\pm \mathrm{BT} \uparrow$

- Thrombocytopenia

- Infection, ITP, Leukemia

- Bleeding time $\uparrow$

- vWF

- Normal

- EDS

- Platelet aggregation defect eg. Medications, Glanzman thrombasthenia, Bernard Soulier syndrome

- Abnormal

- Vwd

- PTT个

- Repeat after 1:1 mix with plasma

- PTT corrects

- Yes

- vWF activity normal

- vWD

- vWF activity normal

- Factors VIII,IX, XI, XII

- Hemophilia A,B,C,

- Hageman Factor Deficiency

- No

- Heparin inhibitors,

- SLE
- 个PT

- Repeat after 1:1 mix with plasma

- PTT corrects

- No

- Lymphomas

- Penicillin

- Post viral infections

- Yes

- Vit K trial

- PT not corrected

- Assay factors VII, II, V, X

- Deficiency of VII, II, V,X

- PT corrected

- Vit K def

- Dicumarol

- Warfarin

- TT $_{\mathrm{TT}}$

- Uremia

- Liver disease

- Dysfibrigenomia

- Hypofibrigenemia

- Cong afbrinogenemia

- 个PTT

- 个PT

- $\uparrow T T$

- $\downarrow$ Fibrinogen

- DIC

- Liver disease

- Normal Lab screening

- Child abuse

- EDS

- Hereditary hemorrhagic telangiectasia

- Factor XIII def

- Vasculitis

- Scurvy

Figure 3: The algorithm for stepwise approach to diagnose bleeding disorder. ${ }^{13 a, 30}$ 


\section{Conclusion:}

Because of the facts that multiple systems might get involved with a bleeding disorder and the relations among the intricately related systems are such that the evaluation always demands some critical analyses of history, examination and laboratory results as discussed above. To make the solution straight and simple one should look into the problem of bleeding disorder either from Platelet or from Coagulation defect or from the vascular pathology. Examining the Coagulation defects should focus either on Extrinsic Pathway or the Intrinsic or the Common Pathway. So, by gaining the ideas and clues of the facts the specific test can finalize the diagnosis.

\section{References:}

1. Lee A. Emergency management of the patients with bleeding disorders: Practical points for the emergency physicians. Transfusion and Apheresis Science. 2019;58(5):553-562.

2. Scott JP, Flood VH, Raffini LJ. Hemostasis. In Chapter 502. Nelson Textbook of Pediatrics. 21st edition. Eds, Kliegman RM, Stanton BF, Schor NF, St. Geme JW, Behrman RE. Saunders. Philadelphia. 2019: 2589-2594.

3. Colman RW et al. Overview of Hemostasis. In: Colman RW et al. eds. Hemostasis and Thromosis, 4th ed. Philadelphia: Lippincott Williams \& Wilkins. 2001:3-16.

4. Blanco A, Blanco G. Hemostasis. Medical Biochemistry. Chapter 31. Academic Press.2017:781-789.

5. Gale AJ. Current understanding of hemostasis. ToxicolPathol.2011;38(1):273-280.

6. Kenichi AT, Nigel SK, Jerold HL. Blood Coagulation: Hemostasis and thrombin regulation. Anesthesia and Analgesia.2009;108:1433-46.

7. Nicpon E; Katja H. Human Anatomy \& Physiology (8th ed.). San Francisco:Benjamin Cummings.2010:649-50.

8. Boon, G. D. "An Overview of Hemostasis." Toxicologic Pathology. 1993; 21(2): 170-79.

9. Alturi, Pavan. The Surgical Review: An Integrated Basic and Clinical Science Study Guide. Philadelphia: Lippincott Williams \& Wilkins.2005; pp. 300.
10. Zdanowicz, M. Essentials of pathophysiology for pharmacy. Florida: CRC Press. 2003; pp. 23.

11. Zhenyu Li. "Signaling during platelet adhesion and activation". Arteriosclerosis, Thrombosis, and Vascular Biology. 2010; 30: 2341-2349. doi: 10.1161/ ATVBAHA. 110.207522. PMC 3085271. PMID 21071698.

12. Sanjeev $P$, Richa $S$, Anshu $P$. Overview of the coagulation system. Indian Journal of Anesthesia. 2014;58(5):516-523.

13. Catherin PMH, Karen AM, Yang L, Laboratory Investigations for Bleeding Disorders. Sembin Thromb Hemost. 2012; 38: 742-752.

13a. Suchitra SA, Susmita NS.Disorders of Coagulation. Chapter 15. In: Lanzkowsky's Manual of Pediatric Hematology and Oncology 6th edition Eds. Lanzkowsky P, Lipton JM, Fish JD. Mica Haley, London.2016:279-333.

14. Johnson M, Zipursky A. Microtechnology for the study of of the blood coagulation system in the newborn infants. Can J Med Tech. 1980;42:159164.

15. Andrew M, Paes B, Milner R, et al. Development of the coagulation system in the full term infant. Blood 1987. 70:165-72.

16. Andrew M, Paes B, Milner $R$ et al. Development of the coagulation system in the healthy prepature infant. Blood. 1988;72:1651-77.

17. Andrew M, Paes B, Milber $R$ et al. Development of the hemostatic system in the neonate and young infant. $\mathrm{Am} J$ Pediiatr Hematol and Oncol.1990;12:95-104.

18. Corrigan JJ Jr. Normal Hemostasis in fetus and newborn. Coagulation:InPolin RA, Fox WW (es)Neonatal and Fetal Medicine, Patholgy and Pathohysiology. Philadelphia: WB Saunders. 1992:1368-1371.

19. Cade JF, Hirsh J, Martin M. Placental barriers to coagulation factors: Its relevance to coagulation defects at birth and haemorrhage in the new born. Br Med J.1969;2:281-283.

20. Katz JA, Moake JL, McPherson PD et al. Relationship between human development and disappearance of unusually large von Willebrand factor multimers from plasma. Blood. 1989;18511858. 
21. Weinstein MJ, Blanchard R, Moake JL, Vosburgh E, Moise K. Fetal and neonatal von Willebrand Factor (VWF) is unusually large and similar to the $V W F$ in patients with thrombotic thrombocytopenic purpura. $\mathrm{Br} J$ Haematol. 1989;72:68-72.

22. Ts'ao $\mathrm{CH}$, Green $\mathrm{D}$, Schultz K. Function and ultrastructure of platelets of neonates: enhanced ristocetin aggregation of neonatal platelets. $\mathrm{Br} \mathrm{J}$ Haematol. 1976;32:225-233.

23. Greffe BS, Marlar RA, Manco-Johnson $M$. Neontal protein C: Molecular composition and distribution in normal term infants. Thromb Res. 1989;56:91-98.

24. Greffe BS, Manco-Johnson MJ, Marlar RA. Molecular forms of human C:Comparison and distribution in human adult plasma. ThrombHaemostas. 1989;62:902-95.

25. Scwartz HP, Muntean W, Watzke H, Richter B, Griffin $\mathrm{JH}$. Low total protein $S$ antigen but high protein $S$ activity due to decreased C4b-binding protein in neonates. Blood.1988;71:562-565.

26. Moalic $P$, Gruel $Y$, Body $G$, Foloppe $P$, Dalahouse B, Leroy J. Levels and plasma distribution of free and $C 4 b$-bound protein $S$ in human foetuses and full term newborns. Thrombo Res. 1988;49:471-480.

27. Warr TA, Warn-Crammer BJ, Rao LVM, Rapaport SI. Human plasma extrinsic pathway inhibitor activity: Standardization of assay and evaluation of physiological variables. Blood 1989;74: 201-206.

28. Weissbach G, Harenberg J, Wendisch J, Pargac $N$, Thomas $K$. Tissue factor pathway inhibitor in infants and children. Throm Res 1994;73:441446.

29. Corrigan JJ, Sluth JJ, Jetter $M$ et al. Newbornsfibrinolyticmechanism: Components and plasmin generation. Am $J$ Hematol. 1989;32:273-278.

30. Al-Huniti A, Sharathkumar A, Manwani D. Approach to the PediatricPatien with a bleeding disorder. Chapter 32 In:Hemostaasis and Bleeding Disorder 3rd edition. Eds. Beth HS, Christopher D, Hillyer CD, Morayma RG. Elsevier Inc. 2019:565-570. 